\title{
ON WARING'S PROBLEM FOR CUBES AND SMOOTH WEYL SUMS
}

\author{
JÖRG BRÜDERN and TREVOR D. WOOLEY
}

[Received 8 May 1999; revised 27 July 1999 and 24 October 1999]

\section{Introduction}

Investigations concerning sums of cubes occupy a distinguished place in the theory of Waring's problem, owing to the special interest associated with additive problems of smaller degree. As a consequence of extensive investigations it is known, for example, that all large integers are the sum of seven cubes of natural numbers (Linnik [7]), that the expected asymptotic formula holds for the number of representations of a large integer as the sum of eight cubes of natural numbers (Vaughan [9]), and that all large integers in the expected congruence classes are the sum of nine cubes of prime numbers (see Theorem 11 of Hua [6]). The new iterative methods most recently associated with the use of exponential sums over smooth numbers (that is, numbers possessing only small prime factors), which have provided the foundation for substantial progress on Waring's problem in the large (see Vaughan [10] and Wooley [13]), have wrought flexible new tools for the investigation of additive problems involving cubes. Such methods played a role, for example, in the recent proof that all large integers are the sum of seven cubes of almost primes (see Brüdern [3]; here 'almost prime' means an integer with at most 69 prime factors, counted with multiplicity). It is therefore somewhat ironic that progress on the analogue of Waring's problem for sums of cubes of smooth numbers has been decidedly modest. The strongest conclusion available hitherto is that all large integers are the sum of nine cubes of smooth numbers (see Harcos [5]). The object of this paper is to establish a similar result with but eight cubes. In order to establish such a conclusion, we are forced to develop the theory of cubic smooth Weyl sums in several new directions, and our results here will be of interest beyond the present application (by way of illustration see, for example, Brüdern and Wooley [4]). Moreover many of the ideas described herein may be adapted easily for use in cognate problems associated with $k$ th powers for $k$ exceeding 3 .

Before recording our main theorem, which we establish in $\S 5$ below, it is convenient to describe some notation. When $n$ is a positive integer, we write $P(n)$ for the largest prime factor of $n$.

THEOREM 1. There are fixed positive real numbers, $c_{1}$ and $c_{2}$, with the property that whenever the positive integer $n$ is sufficiently large, then the number

\footnotetext{
The second author is a Packard Fellow, and supported in part by NSF grant DMS-9622773. He is grateful to the Mathematisches Institut A at Stuttgart for its generous hospitality during the period in which this paper was written.

2000 Mathematics Subject Classification: 11P05, 11L15, 11P55.

Proc. London Math. Soc. (3) 82 (2001) 89-109. (C) London Mathematical Society 2001
} 
of positive integral solutions of the equation

with

$$
m_{1}^{3}+\ldots+m_{8}^{3}=n
$$

exceeds

$$
P\left(m_{i}\right) \leqslant \exp \left(c_{1}(\log n \log \log n)^{1 / 2}\right) \quad(1 \leqslant i \leqslant 8)
$$

$$
n^{5 / 3} \exp \left(-c_{2}(\log n \log \log n)^{1 / 2}\right) .
$$

For comparison, the main theorem of Harcos [5] provides a similar conclusion wherein the number of summands 8 above is replaced by 9. As experts will recognise, the methods employed in our proof of Theorem 1 permit one to establish that almost all positive integers are the sum of four positive integral cubes, the largest prime divisors of which are small in the sense described in the statement of Theorem 1. We discuss such matters briefly at the end of $\S 5$.

Our proof of Theorem 1 makes critical use of the new knowledge concerning upper bounds for fractional moments of smooth Weyl sums provided by 'breaking classical convexity' (see Wooley [14]). The mean value estimates obtained through this new approach may be of use elsewhere, and so in $\S 4$ we provide detailed data associated with such upper bounds. A consequence of this work which has already found application beyond this paper (see Brüdern and Wooley [4]) merits announcement at this point, and this requires further notation. When $P$ and $R$ are positive real numbers, we define the set $\mathscr{A}(P, R)$ of $R$-smooth numbers of size at most $P$ by

$$
\mathscr{A}(P, R)=\{n \in[1, P] \cap \mathbb{Z}: p \mid n \text { and } p \text { prime } \Rightarrow p \leqslant R\} .
$$

We define also the smooth Weyl sum $f(\alpha ; P, R)$ by

$$
f(\alpha ; P, R)=\sum_{x \in \mathscr{A}(P, R)} e\left(\alpha x^{3}\right),
$$

where as usual we write $e(z)$ for $e^{2 \pi i z}$. In circumstances where confusion is easily avoided, we abbreviate $f(\alpha ; P, R)$ to $f(\alpha)$, and likewise for any functions associated with $f(\alpha ; P, R)$.

THEOREM 2. There exist positive numbers $\eta$ and $c$ with the following property. Whenever $P$ and $R$ are real numbers with $P$ sufficiently large in terms of $\eta$, and satisfying

$$
\exp \left(c(\log \log P)^{2}\right) \leqslant R \leqslant P^{\eta},
$$

then for each real number $s$ with $s \geqslant 7.691$ one has

$$
\int_{0}^{1}|f(\alpha ; P, R)|^{s} d \alpha \ll P^{s-3} .
$$

On consideration of the underlying diophantine equation, a conclusion of the type provided by Theorem 2 is immediate from Vaughan [9, Theorem 2] for $s \geqslant 8$, but apparently no such estimate was available hitherto for $s<8$. When there is a positive number $\eta_{0}$ with the property that $R \gg P^{\eta_{0}}$, then familiar estimates (see, for example, Chapter 12 of Vaughan [11]) provide the lower bound $\operatorname{card}(\mathscr{A}(P, R)) \gg P$. In such circumstances, therefore, one readily establishes the 
lower bound

$$
\int_{0}^{1}|f(\alpha ; P, R)|^{s} d \alpha \gg P^{s-3} .
$$

It follows that Theorem 2 provides an estimate of the correct order of magnitude, although one should emphasise that one expects this estimate to hold for $s \geqslant 6$.

In order to establish Theorems 1 and 2 we apply the Hardy-Littlewood method. Our first step in $\S 2$ is to prepare estimates of use on the minor arcs of the dissection, the conventional estimates for Weyl sums being unavailable in the current context. Fortunately, rather general work of Balog and Brüdern [2] concerning cubic exponential sums leads comfortably to suitable estimates. The traditional approaches to estimating the major arc contribution from a treatment involving smooth Weyl sums rely either on the presence of complete Weyl sums to gain the upper hand, or else on the presence of a multitude of smooth Weyl sums in order that possible wild behaviour be swamped. Neither situation pertaining to the situation at hand, we are forced in $\S 3$ to develop a new method of analysing the major arc contribution from a product of smooth Weyl sums. This treatment will be of interest beyond the present context. Next, in $\S 4$, we discuss fractional moments of smooth Weyl sums, concluding with the proof of Theorem 2. Fully equipped at last, in $\S 5$ we are finally able to complete the proof of Theorem 1 .

Throughout, $\varepsilon$ will denote a sufficiently small positive number. We use $\ll$ and $\gg$ to denote Vinogradov's well-known notation, with implicit constants depending at most on $\varepsilon$. In an effort to simplify our analysis, we adopt the following convention concerning the number $\varepsilon$. Whenever $\varepsilon$ appears in a statement, either implicitly or explicitly, we assert that for each $\varepsilon>0$, the statement holds for sufficiently large values of the main parameter. Note that the 'value' of $\varepsilon$ may consequently change from statement to statement, and hence also the dependence of implicit constants on $\varepsilon$. Finally, when $y$ is a real number we write $[y]$ for the greatest integer not exceeding $y$.

The authors thank the referee for careful comments.

\section{Minor arc estimates}

We begin our investigations by furnishing ourselves with a minor arc estimate for the smooth Weyl sum $f(\alpha ; P, R)$. Although the use of rather general estimates (see Vaughan [10, Theorem 1.8]) would suffice for a weak estimate adequate to establish Theorem 1, our aim here is to obtain an estimate as strong as possible in order that Theorem 2 be applicable in the widest range feasible. Before proceeding further we require some notation.

We write $d(q)$ for the number of divisors of the integer $q$. Also, we let $\kappa(q)$ denote the multiplicative function defined on prime powers by taking

$$
\kappa\left(p^{3 l}\right)=p^{-l}, \quad \kappa\left(p^{3 l+1}\right)=2 p^{-l-1 / 2}, \quad \kappa\left(p^{3 l+2}\right)=p^{-l-1},
$$

for $l$ a non-negative integer. When $L$ and $R$ are positive real numbers and $\pi$ is a prime number, we define the set of smooth numbers $\mathscr{B}(L, \pi, R)$ by

$$
\mathscr{B}(L, \pi, R)=\{x \in \mathbb{N}: L<x \leqslant L \pi, \pi|x, p| x \text { and } p \text { prime } \Rightarrow \pi \leqslant p \leqslant R\},
$$


and we define an associated set $\mathscr{B}^{*}(L, \pi, R)$ by

$$
\mathscr{B}^{*}(L, \pi, R)=\{y \in \mathbb{N}: y \pi \in \mathscr{B}(L, \pi, R)\} .
$$

Let $\pi$ be a prime number and let $P, M$ and $R$ be positive real numbers to be fixed later. Define the sequences $\left(a_{m}\right)$ and $\left(b_{n}\right)$ by

$$
a_{m}= \begin{cases}1, & \text { when } m \in \mathscr{B}^{*}(M, \pi, R), \\ 0, & \text { otherwise }\end{cases}
$$

and

$$
b_{n}= \begin{cases}1, & \text { when } n \in \mathscr{A}(P, \pi), \\ 0, & \text { otherwise. }\end{cases}
$$

Finally, when $\pi$ is a prime number and $U$ and $X$ are positive real numbers, we write

$$
S_{\pi}(\alpha ; U, X)=\sum_{U<u \leqslant 2 U} \sum_{X<u v \leqslant 2 X} a_{u} b_{v} e\left(\alpha(\pi u v)^{3}\right) .
$$

It is convenient at this stage to record an estimate which is immediate from work of Balog and Brüdern [2, Lemma 5].

Lemma 2.1. Suppose that $U \geqslant 11 X^{2 / 3}$. Then whenever $b \in \mathbb{Z}$ and $r \in \mathbb{N}$ satisfy $(b, r)=1, r \leqslant \frac{1}{2} U^{9 / 4}$ and $\left|r \alpha \pi^{3}-b\right| \leqslant 2 U^{-9 / 4}$, one has

$$
\left|S_{\pi}(\alpha ; U, X)\right|^{2} \ll X U+X^{2} U^{\varepsilon-1 / 4}+\frac{\kappa(r) d(r)^{4} X^{2} \log X}{1+X^{3}\left|\pi^{3} \alpha-b / r\right|} .
$$

We are now prepared to establish our minor arc estimate for cubic smooth Weyl sums, which we record in the following lemma.

Lemma 2.2. Let $\delta$ be any real number with $\frac{3}{5}<\delta<\frac{12}{5}$, let $\varepsilon$ be a positive number sufficiently small in terms of $\delta$, and let $\eta$ be a positive number sufficiently small in terms of $\delta$ and $\varepsilon$. Let $P$ be a real number sufficiently large in terms of $\delta$, $\varepsilon$ and $\eta$, and let $R$ be a real number with $2 \leqslant R \leqslant P^{\eta}$. Suppose that $\alpha$ is a real number, and suppose also that $a \in \mathbb{Z}$ and $q \in \mathbb{N}$ satisfy $(a, q)=1, q \leqslant P^{3-\delta}$ and $|q \alpha-a| \leqslant P^{\delta-3}$. Then

$$
f(\alpha ; P, R) \ll \frac{q^{\varepsilon}(\kappa(q))^{1 / 2} P(\log P)^{5 / 2+\varepsilon}}{\left(1+P^{3}|\alpha-a / q|\right)^{1 / 3}}+P^{9 / 10+\varepsilon} .
$$

Proof. We begin by fixing $M=P^{4 / 5}$, and note that Lemma 10.1 of Vaughan [10] shows that for each $w \in \mathscr{A}(P, R)$ with $w>M$, there exists a unique triple $(\pi, u, v)$ with $w=u v, \pi$ prime, $u \in \mathscr{B}(M, \pi, R)$ and $v \in \mathscr{A}(P / u, \pi)$. Consequently, on recalling the definitions (1.1) and (2.2), one has

$$
\begin{aligned}
f(\alpha) & =\sum_{\substack{w \in \mathscr{A}(P, R) \\
w>P^{9 / 10}}} e\left(\alpha w^{3}\right)+O\left(P^{9 / 10}\right) \\
& =\sum_{\pi \leqslant R} \sum_{\substack{i=0 \\
2^{i} \leqslant \pi}}^{\infty} \sum_{\substack{j=0 \\
P^{9 / 10}<2^{j} \pi \leqslant P}}^{\infty} S_{\pi}\left(\alpha ; 2^{i} M \pi^{-1}, 2^{j}\right)+O\left(P^{9 / 10}\right) .
\end{aligned}
$$


Next consider a prime number $\pi$ and real numbers $U$ and $X$ with $M \pi^{-1}<U \leqslant M$ and $P^{9 / 10}<\pi X \leqslant P$. Our choice of $M$ ensures that $U \geqslant 11 X^{2 / 3}$, provided that $P$ is sufficiently large. By Dirichlet's Theorem on diophantine approximation, there exist $b \in \mathbb{Z}$ and $r \in \mathbb{N}$ with

$$
(b, r)=1, \quad r \leqslant \frac{1}{2} U^{9 / 4} \text { and }\left|r \alpha \pi^{3}-b\right| \leqslant 2 U^{-9 / 4} .
$$

Then on making use of the relations (2.1) together with an elementary estimate for the divisor function, we see from Lemma 2.1 that

$$
\begin{aligned}
\left|S_{\pi}(\alpha ; U, X)\right|^{2} & \ll X U+X^{2} U^{\varepsilon-1 / 4}+\frac{\kappa(r) d(r)^{4} X^{2} \log X}{1+X^{3}\left|\pi^{3} \alpha-b / r\right|} \\
& \ll X U+X^{2} U^{\varepsilon-1 / 4}+\frac{r^{\varepsilon-1 / 3} X^{2} \log X}{1+X^{3}\left|\pi^{3} \alpha-b / r\right|} .
\end{aligned}
$$

We therefore deduce that whenever $r>X^{6+20 \varepsilon} P^{-27 / 5}$, then necessarily one has

$$
\left|S_{\pi}(\alpha ; U, X)\right| \ll P^{9 / 10+\varepsilon} \text {. }
$$

If, on the other hand, one has $r \leqslant X^{6+20 \varepsilon} P^{-27 / 5}$, then the estimate (2.5) again follows whenever

$$
\left|\pi^{3} r \alpha-b\right|>r^{2 / 3+\varepsilon} P^{\varepsilon-9 / 5} X^{-1} \log X
$$

and moreover this inequality is satisfied provided only that

$$
\left|\pi^{3} r \alpha-b\right|>\left(X^{6+20 \varepsilon} P^{-27 / 5}\right)^{2 / 3+\varepsilon} P^{\varepsilon-9 / 5} X^{-1} \log X .
$$

We may therefore conclude that either the inequality (2.5) holds, or else there exist $b \in \mathbb{Z}$ and $r \in \mathbb{N}$ with

$$
(b, r)=1, \quad r \leqslant X^{6+20 \varepsilon} P^{-27 / 5} \text { and }\left|\pi^{3} r \alpha-b\right| \leqslant X^{3+20 \varepsilon} P^{-27 / 5},
$$

and moreover that in the latter circumstance one has

$$
\left|S_{\pi}(\alpha ; U, X)\right| \ll \frac{(\kappa(r))^{1 / 2} d(r)^{2} X(\log X)^{1 / 2}}{\left(1+X^{3}\left|\pi^{3} \alpha-b / r\right|\right)^{1 / 3}} .
$$

Suppose that the latter situation above holds, so that (2.6) and (2.7) hold. On recalling the hypotheses of the statement of the lemma, it follows from the triangle inequality that

Consequently,

$$
\left|\frac{a}{q}-\frac{b}{r \pi^{3}}\right| \leqslant q^{-1} P^{\delta-3}+\left(r \pi^{3}\right)^{-1} X^{3+20 \varepsilon} P^{-27 / 5}
$$

$$
\begin{aligned}
\left|a r \pi^{3}-b q\right| & \leqslant X^{6+20 \varepsilon} P^{\delta-42 / 5} \pi^{3}+X^{3+20 \varepsilon} P^{-12 / 5-\delta} \\
& \leqslant P^{20 \varepsilon+\delta-12 / 5}+P^{20 \varepsilon+3 / 5-\delta}
\end{aligned}
$$

Then our hypotheses concerning $\delta, \varepsilon$ and $\eta$ ensure that $\left|a r \pi^{3}-b q\right|<1$, whence $a r \pi^{3}=b q$. But by hypothesis one has $(a, q)=(b, r)=1$, and thus $r \mid q$ and $q \mid r \pi^{3}$. Thus we find that for some $I$ with $0 \leqslant I \leqslant 3$ one has $q=r \pi^{I}$ and $b=$ $a \pi^{3-I}$. When $\pi \mid q$, it follows from (2.1) and (2.7) that

$$
\left|S_{\pi}(\alpha ; U, X)\right| \ll \frac{(\pi \kappa(q))^{1 / 2} d(q)^{2} X(\log X)^{1 / 2}}{\left(1+(X \pi)^{3}|\alpha-a / q|\right)^{1 / 3}},
$$


and when $\pi \nmid q$ one similarly obtains

$$
\left|S_{\pi}(\alpha ; U, X)\right| \ll \frac{(\kappa(q))^{1 / 2} d(q)^{2} X(\log X)^{1 / 2}}{\left(1+(X \pi)^{3}|\alpha-a / q|\right)^{1 / 3}} .
$$

On combining the conclusions of the above discussion with (2.4), we obtain

$$
\begin{aligned}
f(\alpha) \ll & P^{9 / 10+\varepsilon}+\sum_{\substack{\pi \leqslant R \\
\pi \mid q}}(\log P)^{2} \max _{P^{9 / 10}<X \pi \leqslant P} \frac{(\pi \kappa(q))^{1 / 2} d(q)^{2} X(\log X)^{1 / 2}}{\left(1+(X \pi)^{3}|\alpha-a / q|\right)^{1 / 3}} \\
& +\sum_{\substack{\pi \leqslant R \\
\pi \nmid q}}(\log P)^{2} \max _{P^{9 / 10}<X \pi \leqslant P} \frac{(\kappa(q))^{1 / 2} d(q)^{2} X(\log X)^{1 / 2}}{\left(1+(X \pi)^{3}|\alpha-a / q|\right)^{1 / 3}} .
\end{aligned}
$$

Thus one deduces that

$$
f(\alpha) \ll P^{9 / 10+\varepsilon}+\frac{d(q)^{2}(\kappa(q))^{1 / 2} P(\log P)^{5 / 2}}{\left(1+P^{3}|\alpha-a / q|\right)^{1 / 3}}\left(\sum_{\pi \mid q} \pi^{-1 / 2}+\sum_{\pi \leqslant R} \pi^{-1}\right),
$$

whence, by elementary prime number theory and an elementary estimate for the divisor function, we finally arrive at the estimate (2.3). This completes the proof of the lemma.

\section{Some major arc estimates}

As is usual in modern applications of the Hardy-Littlewood method, our major arc treatment rests for its success on a suitable pruning lemma. Since this lemma will be applicable in a wider context, it deserves a somewhat abstract formulation. We first require some additional notation. When $X$ is a real number with $1 \leqslant X \leqslant P$, we define the set of major $\operatorname{arcs} \mathfrak{M}(X)$ to be the union of the intervals

$$
\mathfrak{M}(q, a ; X)=\left\{\alpha \in[0,1):|q \alpha-a| \leqslant X P^{-3}\right\}
$$

with $0 \leqslant a \leqslant q \leqslant X$ and $(a, q)=1$. We then define the minor $\operatorname{arcs} \mathfrak{m}(X)$ by taking $\mathfrak{m}(X)=[0,1) \backslash \mathfrak{M}(X)$. Observe that the sets $\mathfrak{M}(q, a ; X)$ comprising $\mathfrak{M}(X)$ are mutually disjoint. Finally, it is useful to adopt the convention of writing $\mathfrak{N}(X)=\mathfrak{M}(2 X) \backslash \mathfrak{M}(X)$.

Lemma 3.1. Recall the multiplicative function $\kappa(q)$ defined by (2.1). Let $Q$ be a real number with $1 \leqslant Q \leqslant P$, and define the function $\Upsilon(\alpha)$ for $\alpha \in \mathfrak{M}(Q)$ by taking

$$
\Upsilon(\alpha)=\kappa(q)^{2}\left(1+P^{3}|\alpha-a / q|\right)^{-1}
$$

when $\alpha \in \mathfrak{M}(q, a ; Q) \subseteq \mathfrak{M}(Q)$. Then there is an absolute constant $B$ such that for any subset $\mathscr{A}$ of $[1, P] \cap \mathbb{Z}$, one has

$$
\int_{\mathfrak{M}(Q)} \Upsilon(\alpha)\left|\sum_{x \in \mathscr{A}} e\left(\alpha x^{3}\right)\right|^{2} d \alpha \ll P^{-1}(\log (2 Q))^{B} .
$$


Proof. Observe first that

$$
\begin{aligned}
\int_{\mathfrak{M}(Q)} \Upsilon(\alpha)\left|\sum_{x \in \mathscr{A}} e\left(\alpha x^{3}\right)\right|^{2} d \alpha \\
\leqslant \sum_{1 \leqslant q \leqslant Q} \kappa(q)^{2} \sum_{\substack{a=1 \\
(a, q)=1}}^{q} \int_{-Q / P^{3}}^{Q / P^{3}}\left(1+P^{3}|\beta|\right)^{-1}\left|\sum_{x \in \mathscr{A}} e\left(x^{3}(\beta+a / q)\right)\right|^{2} d \beta .
\end{aligned}
$$

Let $c_{q}(n)$ be Ramanujan's sum, which we define by

$$
c_{q}(n)=\sum_{\substack{a=1 \\(a, q)=1}}^{q} e(a n / q)
$$

Then it follows that

$$
\sum_{\substack{a=1 \\(a, q)=1}}^{q}\left|\sum_{x \in \mathscr{A}} e\left(x^{3}(\beta+a / q)\right)\right|^{2}=\sum_{x, y \in \mathscr{A}} c_{q}\left(x^{3}-y^{3}\right) e\left(\beta\left(x^{3}-y^{3}\right)\right) .
$$

But on making the convention that $(q, 0)=q$, one has the well-known estimate $\left|c_{q}(n)\right| \leqslant(q, n)$, whence (3.1) yields

$$
\int_{\mathfrak{M}(Q)} \Upsilon(\alpha)\left|\sum_{x \in \mathscr{A}} e\left(\alpha x^{3}\right)\right|^{2} d \alpha \ll P^{-3}(\log (2 Q)) \sum_{1 \leqslant q \leqslant Q} \kappa(q)^{2} \sum_{1 \leqslant x, y \leqslant P}\left(q, x^{3}-y^{3}\right) \text {. }
$$

In order to analyse the final summation of (3.2), we introduce the function $\rho(r)$, which when $r$ is a natural number we define to be the number of solutions of the congruence $x^{3} \equiv y^{3}(\bmod r)$ with $1 \leqslant x, y \leqslant r$. For any natural number $r$ one may write $r=r_{1} r_{3}^{3}$ with $r_{1}$ cube-free, and moreover this decomposition is unique. Further, an elementary counting argument shows that, for a suitable fixed positive number $C$ (one may check that $C=8$ is admissible), one has $\rho(r) \ll C^{\omega(r)} r r_{3}$, where here and throughout $\omega(n)$ denotes the number of prime divisors of $n$. Then by sorting the variables $x$ and $y$ into residue classes, one obtains

$$
\begin{aligned}
\sum_{1 \leqslant x, y \leqslant P}\left(q, x^{3}-y^{3}\right) & \leqslant \sum_{d \mid q} d \cdot \operatorname{card}\left(\left\{1 \leqslant x, y \leqslant P: x^{3} \equiv y^{3}(\bmod d)\right\}\right) \\
& \leqslant \sum_{d \mid q}\left(\frac{P}{d}+1\right)^{2} d \rho(d)
\end{aligned}
$$

When $q \leqslant P$, therefore, we deduce that

$$
\sum_{1 \leqslant x, y \leqslant P}\left(q, x^{3}-y^{3}\right) \ll P^{2} \sum_{d \mid q} d^{-1} \rho(d) \ll P^{2} \sum_{d \mid q} C^{\omega(d)} d_{3} \ll P^{2} C^{\omega(q)} d(q) q_{3} .
$$

On substituting the estimate (3.3) into (3.2) and recalling the definition (2.1), 
we may conclude that

$$
\begin{aligned}
& \int_{\mathfrak{M}(Q)} \Upsilon(\alpha)\left|\sum_{x \in \mathscr{A}} e\left(\alpha x^{3}\right)\right|^{2} d \alpha \\
& \ll P^{-1}(\log (2 Q)) \sum_{1 \leqslant q \leqslant Q}\left(C^{\omega(q)} d(q) q_{3}\right)\left(4^{\omega\left(q_{1}\right)} q_{1}^{-1} q_{3}^{-2}\right),
\end{aligned}
$$

and thus the elementary theory of arithmetic functions yields the bound

$$
\int_{\mathfrak{M}(Q)} \Upsilon(\alpha)\left|\sum_{x \in \mathscr{A}} e\left(\alpha x^{3}\right)\right|^{2} d \alpha \ll P^{-1}(\log (2 Q))^{B},
$$

for a suitable absolute constant $B$. This completes the proof of the lemma.

As an immediate corollary of Lemma 3.1, we are able to obtain an estimate for the sixth moment of $f(\alpha ; P, R)$, restricted to suitable major arcs, which is close to the best possible such bound.

COROLlaRY 3.2. Let $\delta$ be a real number with $0<\delta<\frac{2}{5}$, let $\varepsilon$ be a small positive number, and let $\eta$ be a positive number sufficiently small in terms of $\delta$ and $\varepsilon$. Let $P$ be a real number sufficiently large in terms of $\delta, \varepsilon$ and $\eta$, and let $R$ be a real number with $2 \leqslant R \leqslant P^{\eta}$. Then whenever $X$ is a real number with $1 \leqslant X \leqslant P^{\delta}$, one has

$$
\int_{\mathfrak{M}(X)}|f(\alpha ; P, R)|^{6} d \alpha \ll X^{\varepsilon} P^{3}(\log P)^{10+\varepsilon} .
$$

Proof. Consider a real number $\alpha \in \mathfrak{M}(q, a ; X) \subseteq \mathfrak{M}(X)$. One has $(a, q)=1$, $q \leqslant X$ and $|q \alpha-a| \leqslant X P^{-3}$, so that since $X \leqslant P^{2 / 5}$, it follows from Lemma 2.2 that the estimate (2.3) holds. An inspection of (2.1) reveals that the expression

$$
\frac{q^{\varepsilon}(\kappa(q))^{1 / 2} P(\log P)^{5 / 2+\varepsilon}}{\left(1+P^{3}|\alpha-a / q|\right)^{1 / 4}}
$$

dominates the right-hand side of (2.3) whenever $\alpha \in \mathfrak{M}(q, a ; X) \subseteq \mathfrak{M}(X)$. Thus we deduce that

$$
\int_{\mathfrak{M}(X)}|f(\alpha)|^{6} d \alpha \ll P^{4}(\log P)^{10+\varepsilon} X^{\varepsilon} \int_{\mathfrak{M}(X)} \Upsilon(\alpha)|f(\alpha)|^{2} d \alpha,
$$

where $\Upsilon(\alpha)$ is the function defined in the statement of Lemma 3.1. But as an immediate consequence of Lemma 3.1,

$$
\int_{\mathfrak{M}(X)} \Upsilon(\alpha)|f(\alpha)|^{2} d \alpha \ll X^{\varepsilon} P^{-1}
$$

whence the conclusion of the lemma is immediate from (3.4).

We remark that the factor $X^{\varepsilon}$ occurring in the statement of Corollary 3.2 may be replaced by the factor $(\log (2 X))^{A}$, for a suitable fixed real number $A$. All that is necessary is a more careful analysis of the arithmetic functions underlying the terms involving $q^{\varepsilon}$ in the above argument, and the use of estimates from the 
elementary theory of arithmetic functions. In particular, therefore, one has an estimate of the type

$$
\int_{\mathfrak{M}(X)}|f(\alpha ; P, R)|^{6} d \alpha \ll P^{3}(\log P)^{A},
$$

for a suitable fixed real number $A$, uniformly for $1 \leqslant X \leqslant P^{\delta}$.

\section{Mean value estimates for smooth Weyl sums}

In this section we discuss the non-trivial estimates for fractional moments of smooth Weyl sums which form a key component of our proof of Theorem 2 . We first require some further notation. Let $P$ and $R$ be positive real numbers, and recall the definition (1.1). When $s$ is a real number, we define the mean value $U_{s}(P, R)$ by

$$
U_{s}(P, R)=\int_{0}^{1}|f(\alpha ; P, R)|^{s} d \alpha .
$$

We say that an exponent $\mu_{s}$ is permissible whenever the exponent has the property that, for each $\varepsilon>0$, there exists a positive number $\eta=\eta(\varepsilon, s)$ such that whenever $R \leqslant P^{\eta}$, one has

$$
U_{s}(P, R) \ll_{\varepsilon, s} P^{\mu_{s}+\varepsilon} .
$$

It is a fact (see Wooley [14]) that for every positive number $s$, one has $\mu_{s} \geqslant \max \left\{\frac{1}{2} s, s-3\right\}$. Moreover, in view of the trivial estimate for $U_{s}(P, R)$, one may always take $\mu_{s} \leqslant s$ for each $s$. It is convenient to describe an exponent $\delta_{s}$ as associated if the exponent $\mu_{s}=\frac{1}{2} s+\delta_{s}$ is permissible, and to describe an exponent $\Delta_{s}$ as admissible if the exponent $\mu_{s}=s-3+\Delta_{s}$ is permissible.

The ideas underlying the calculation of permissible exponents $\mu_{s}$ are described by Wooley in [14] in some generality, and the particular case of cubic smooth Weyl sums is described more specifically in $\S 2$ of Baker, Brüdern and Wooley [1]. We recall the following lemma.

Lemma 4.1. (i) Let $s$ be a real number with $s>2$, and suppose that $\delta_{s}$ and $\delta_{2 s}$ are associated exponents. Then the exponent $\delta_{s+2}$ is associated, where

$$
\delta_{s+2}=\delta_{s}(1-\theta)+\frac{1}{2} s \theta
$$

and

$$
\theta=\frac{\delta_{2 s}-2 \delta_{s}}{4+\delta_{2 s}-2 \delta_{s}}
$$

(ii) Let $s$ be a real number with $s>2$, and suppose that $\delta_{s}$ and $\delta_{4 s / 3}$ are associated exponents. Then the exponent $\delta_{s+2}$ defined by (4.1) is associated, where now we take

$$
\theta=\frac{1+3 \delta_{4 s / 3}-4 \delta_{s}}{9+3 \delta_{4 s / 3}-4 \delta_{s}}
$$

Proof. This lemma is simply a combination of Lemma 1 and the Corollary to Lemma 2 of Baker, Brüdern and Wooley [1].

We augment this lemma with a special method for estimating $\mu_{6}$. 
LemmA 4.2. Suppose that $\delta_{5}$ and $\delta_{6}$ are associated exponents. Then the exponent $\delta_{6}^{\prime}=2 \theta$ is associated, where

$$
\theta=\max \left\{\frac{\delta_{6}}{3+\delta_{6}}, \frac{1+2 \delta_{5}+\delta_{6}}{11+2 \delta_{5}+\delta_{6}}\right\}
$$

Proof. This is immediate from Lemma 5.2 of Wooley [14].

A convexity argument provides effortless bounds not without value.

Lemma 4.3. Suppose that $s>2$ and that $t<s$. Whenever $\delta_{s-t}$ and $\delta_{s+t}$ are associated exponents, then the exponent $\delta_{s}=\frac{1}{2}\left(\delta_{s+t}+\delta_{s-t}\right)$ is also associated.

Proof. Under the hypotheses of the statement of the lemma, an application of Schwarz's inequality yields

$$
\begin{aligned}
\int_{0}^{1}|f(\alpha)|^{s} d \alpha & \ll\left(\int_{0}^{1}|f(\alpha)|^{s-t} d \alpha\right)^{1 / 2}\left(\int_{0}^{1}|f(\alpha)|^{s+t} d \alpha\right)^{1 / 2} \\
& \ll P^{\left(\mu_{s-t}+\mu_{s+t}\right) / 2+\varepsilon} .
\end{aligned}
$$

The lemma is now immediate.

When $s$ is comparatively large, one may obtain new permissible exponents by applying the Hardy-Littlewood method in combination with the analyses of $\S \S 2$ and 3. In preparation for a discussion of such an approach, we require more precise information concerning the behaviour of the smooth Weyl sum $f(\alpha ; P, R)$ on suitable major arcs.

Lemma 4.4. Let $A$ be a positive real number, and let $P$ and $R$ be large real numbers. There are absolute constants $c_{1}$ and $c_{2}$ such that if

$$
\exp \left(c_{1}(\log \log P)^{2}\right) \leqslant R \leqslant P^{1 / 2},
$$

and $a \in \mathbb{Z}$ and $q \in \mathbb{N}$ satisfy $(a, q)=1$ and $q \leqslant(\log P)^{A}$, then for each $\varepsilon>0$ one has

$$
f(\alpha ; P, R) \ll \frac{P}{\left(q+P^{3}|q \alpha-a|\right)^{1 / 3-\varepsilon}}+P \exp \left(-c_{2} \sqrt{\log P}\right)\left(1+P^{3}|\alpha-a / q|\right) .
$$

Proof. If one were to have $R=P^{\eta}$ with $0<\eta<\frac{1}{2}$, then the conclusion of the lemma would be essentially immediate from Vaughan and Wooley [12, Lemma 8.5], but in the present circumstances we must work harder. Suppose then that $\varepsilon$ is a sufficiently small positive number, and that $(\log P) /(\log R)$ is sufficiently large in terms of $\varepsilon$. We begin by observing that in the course of the proof of Lemma 8.5 of [12], the hypothesis described at the start of this proof is not essential for the use of [12, Lemma 8.3], and indeed the hypotheses of the present lemma suffice. Moreover, the aforementioned hypothesis plays an important role only in the preamble to equation (8.6) of [12], wherein the error term should be replaced by a term

$$
O\left(\frac{Q \log (Q / R)}{\log R}\left(1+Q^{k}|\gamma|\right)^{-1 / k}\right)
$$


In the present context, therefore, the argument of the proof of [12, Lemma 8.5] leads to the conclusion that

$$
f(\alpha) \ll \frac{q^{\varepsilon} P \nu}{\left(q+P^{3}|q \alpha-a|\right)^{1 / 3}}+P \exp \left(-c_{2}(\log P)^{1 / 2}\right)\left(1+P^{3}|\alpha-a / q|\right),
$$

where

$$
\nu=(\log P) /(\log R) .
$$

Next observe that by combining an elementary estimate with Theorem 1 of Tenenbaum [8, Chapter III.5], one finds that uniformly for $2 \leqslant R \leqslant P$, one has

$$
\operatorname{card}(\mathscr{A}(P, R)) \ll P e^{-\nu / 2} \text {, }
$$

where $\nu$ is again given by (4.3). Thus for each fixed $N>0$, it follows from (4.2) that

$$
\begin{aligned}
|f(\alpha)|^{N+1} \ll & \frac{q^{N \varepsilon} P^{N+1} \nu^{N} e^{-\nu / 2}}{\left(q+P^{3}|q \alpha-a|\right)^{N / 3}} \\
& +P^{N+1} \exp \left(-N c_{2}(\log P)^{1 / 2}\right)\left(1+P^{3}|\alpha-a / q|\right)^{N} .
\end{aligned}
$$

We take $N=1 / \varepsilon$ and observe that since $\nu$ is sufficiently large in terms of $N$, we may suppose that $\nu^{N} e^{-\nu / 2}<1$. Consequently,

$$
|f(\alpha)| \ll \frac{q^{\varepsilon} P}{\left(q+P^{3}|q \alpha-a|\right)^{1 / 3-\varepsilon}}+P \exp \left(-\frac{1}{2} c_{2}(\log P)^{1 / 2}\right)\left(1+P^{3}|\alpha-a / q|\right) .
$$

The conclusion of the lemma follows immediately.

Lemma 4.5. Let $s$ be a real number with $s \geqslant 6$, and suppose that the exponent $\Delta_{s}$ is admissible. Suppose also that $u$ is a real number with $u>s+10 \Delta_{s}$. Then there exist positive numbers $\eta$ and $c$, depending at most on $u$, with the following property. Whenever $P$ and $R$ are real numbers with $P$ sufficiently large in terms of $\eta$, and satisfying

then one has

$$
\exp \left(c(\log \log P)^{2}\right) \leqslant R \leqslant P^{\eta}
$$

$$
\int_{0}^{1}|f(\alpha ; P, R)|^{u} d \alpha \ll P^{u-3} .
$$

In particular, the exponent $\mu_{w}=w-3$ is permissible for $w \geqslant u$.

Proof. We apply the Hardy-Littlewood method. Let $u$ be a real number satisfying the hypotheses of the statement of the lemma. Let

$$
Q=P^{4 / 5} \text { and } Y=(\log P)^{80 /(u-6)+32},
$$

and write $\mathfrak{m}=\mathfrak{m}(Q), \mathfrak{M}=\mathfrak{M}(Q)$ and $\mathfrak{P}=\mathfrak{M}(Y)$. Suppose that $\alpha \in \mathfrak{m}$. By Dirichlet's Theorem on diophantine approximation, there exist $a \in \mathbb{Z}$ and $q \in \mathbb{N}$ with $(a, q)=1, q \leqslant Q^{-1} P^{3}$ and $|q \alpha-a| \leqslant Q P^{-3}$. Since $\alpha \in \mathfrak{m}$, moreover, one necessarily has $q>Q$. On recalling the relations (2.1), therefore, Lemma 2.2 reveals that

$$
|f(\alpha)| \ll \frac{q^{\varepsilon-1 / 6} P(\log P)^{5 / 2+\varepsilon}}{\left(1+P^{3}|\alpha-a / q|\right)^{1 / 3}}+P^{9 / 10+\varepsilon} \ll P^{9 / 10+\varepsilon} .
$$


Consequently,

$$
\begin{aligned}
\int_{\mathfrak{m}}|f(\alpha)|^{u} d \alpha & \ll\left(\sup _{\alpha \in \mathfrak{m}}|f(\alpha)|\right)^{u-s} \int_{0}^{1}|f(\alpha)|^{s} d \alpha \\
& \ll\left(P^{9 / 10+\varepsilon}\right)^{u-s} P^{s-3+\Delta_{s}+\varepsilon} .
\end{aligned}
$$

But by hypothesis one has $u-s>10 \Delta_{s}$, whence

$$
\Delta_{s}-\frac{1}{10}(u-s)+u \varepsilon<0,
$$

and thus we deduce that

$$
\int_{\mathfrak{m}}|f(\alpha)|^{u} d \alpha \ll P^{u-3} .
$$

Next define the function $f^{*}(\alpha)=f^{*}(\alpha ; P, R)$ for $\alpha \in \mathfrak{M}$ by taking

$$
f^{*}(\alpha ; P, R)=\frac{q^{\varepsilon}(\kappa(q))^{1 / 2} P(\log P)^{5 / 2+\varepsilon}}{\left(1+P^{3}|\alpha-a / q|\right)^{1 / 3}},
$$

when $\alpha \in \mathfrak{M}(q, a ; Q) \subseteq \mathfrak{M}$. Then it follows from Lemma 2.2 that for $\alpha \in \mathfrak{M}$ one has

Write

$$
|f(\alpha)|^{u-s} \ll\left(P^{9 / 10+\varepsilon}\right)^{u-s}+\left|f^{*}(\alpha)\right|^{u-s} .
$$

$$
I_{1}=\int_{\mathfrak{M} \backslash \mathfrak{P}}\left|f^{*}(\alpha)\right|^{u-s}|f(\alpha)|^{s} d \alpha
$$

and

$$
I_{2}=\int_{\mathfrak{P}}|f(\alpha)|^{u} d \alpha .
$$

Then it follows from (4.6) that

$$
\int_{\mathfrak{M}}|f(\alpha)|^{u} d \alpha \ll\left(P^{9 / 10+\varepsilon}\right)^{u-s} \int_{0}^{1}|f(\alpha)|^{s} d \alpha+I_{1}+I_{2},
$$

whence, as in the analysis concluding the treatment of the minor arc contribution,

$$
\int_{\mathfrak{M}}|f(\alpha)|^{u} d \alpha \ll P^{u-3}+I_{1}+I_{2} .
$$

In view of our hypotheses concerning $R$, and the definition of $\mathfrak{P}$, it follows from Lemma 4.4 that when $1 \leqslant X \leqslant Y$ one has

$$
\sup _{\alpha \in \mathfrak{N}(X)}|f(\alpha)| \ll P X^{\varepsilon-1 / 3} .
$$

Since the measure of $\mathfrak{N}(X)$ is $O\left(X^{2} P^{-3}\right)$, it follows that when $1 \leqslant X \leqslant Y$ one has the estimate

$$
\int_{\mathfrak{R}(X)}|f(\alpha)|^{u} d \alpha \ll P^{u-3} X^{2-u(1 / 3-\varepsilon)} .
$$

But $u>6$, so that on summing over dyadic intervals we deduce from (4.8) that

Write

$$
I_{2} \ll P^{u-3} \text {. }
$$

$$
I_{3}=\int_{\mathfrak{M} \backslash \mathfrak{B}}\left|f^{*}(\alpha)\right|^{u-2}|f(\alpha)|^{2} d \alpha
$$


Then on collecting together (4.4), (4.7), (4.9) and (4.10), and applying Hölder's inequality, we obtain

$$
\begin{aligned}
\int_{0}^{1}|f(\alpha)|^{u} d \alpha & =\int_{\mathfrak{m}}|f(\alpha)|^{u} d \alpha+\int_{\mathfrak{M}}|f(\alpha)|^{u} d \alpha \\
& \ll P^{u-3}+\left(\int_{0}^{1}|f(\alpha)|^{u} d \alpha\right)^{(s-2) /(u-2)} I_{3}^{(u-s) /(u-2)},
\end{aligned}
$$

whence

$$
\int_{0}^{1}|f(\alpha)|^{u} d \alpha \ll P^{u-3}+I_{3}
$$

On recalling (2.1) and (4.5), we find that for each real number $X$ with $1 \leqslant X \leqslant Q$, one has

$$
\sup _{\alpha \in \mathfrak{N}(X)}\left|f^{*}(\alpha)\right| \ll P(\log P)^{5 / 2+\varepsilon} X^{-1 / 7} .
$$

Then by (4.5), it follows that

$$
\begin{aligned}
\int_{\mathfrak{N}(X)}\left|f^{*}(\alpha)\right|^{u-2}|f(\alpha)|^{2} d \alpha & \\
& \ll\left(P(\log P)^{5 / 2+\varepsilon} X^{-1 / 7}\right)^{u-6} \int_{\mathfrak{M}(2 X)}\left|f^{*}(\alpha)^{4} f(\alpha)^{2}\right| d \alpha \\
& \ll\left(P(\log P)^{5 / 2+\varepsilon} X^{-1 / 7}\right)^{u-6} P^{4}(\log P)^{10+\varepsilon} X^{\varepsilon} \int_{\mathfrak{M}(2 X)} \Upsilon(\alpha)|f(\alpha)|^{2} d \alpha,
\end{aligned}
$$

where $\Upsilon(\alpha)$ is the function defined in the statement of Lemma 3.1. Thus, by Lemma 3.1 one has

$$
\int_{\mathfrak{N}(X)}\left|f^{*}(\alpha)\right|^{u-2}|f(\alpha)|^{2} d \alpha \ll P^{u-3}(\log P)^{10+3(u-6)} X^{-(u-6) / 8} .
$$

On summing over dyadic intervals, therefore, we may conclude that

$$
I_{3} \ll P^{u-3}(\log P)^{3 u-8} Y^{-(u-6) / 8} \ll P^{u-3} .
$$

Thus, in view of (4.11), we have established the conclusion of the lemma.

One may calculate strong associated exponents using a computer to apply Lemmata 4.1, 4.2, 4.3 and 4.5 as follows. One sets up an array of known associated exponents $\delta_{j h}(0 \leqslant j \leqslant J)$, for some step size $h>0$ and upper limit $J$ (with $J \geqslant 16 / h$ ), by using known bounds for $\delta_{s}$. Thus we have the associated exponents $\delta_{4}=0$ and $\delta_{s}=\frac{1}{2} s-3(s \geqslant 8)$ which follow from Hua's Lemma (see Vaughan [11, Lemma 2.5]), on considering the underlying diophantine equations. In combination with the associated exponent $\delta_{6}=\frac{1}{4}$ due to Vaughan [10, Theorem 4.4], one may apply convexity to provide the associated exponents

$$
\delta_{s}= \begin{cases}0, & \text { when } 0 \leqslant s \leqslant 4, \\ \frac{1}{8}(s-4), & \text { when } 4<s \leqslant 6, \\ \frac{3}{8} s-2, & \text { when } 6<s \leqslant 8, \\ \frac{1}{2} s-3, & \text { when } s>8\end{cases}
$$


For the interesting values of $j$ with $4 / h<j<8 / h$, one may now calculate new associated exponents $\delta_{j h}$ by means of Lemmata 4.1, 4.2, 4.3 and 4.5. Observe that if $\frac{4}{3} j$ is not an integer, but lies between the integers $i$ and $i+1$, then one may apply Lemma 4.1(ii) by use of the convexity bound

$$
\delta_{4 j h / 3} \leqslant\left(i+1-\frac{4}{3} j\right) \delta_{i h}+\left(\frac{4}{3} j-i\right) \delta_{(i+1) h} .
$$

By iterating this process for $4 / h<j<8 / h$, one derives new collections of associated exponents, and eventually this collection converges to some set of limiting values. We note also that Lemma 4.3 may be used in the form

$$
\delta_{j h} \leqslant \frac{1}{2}\left(\delta_{(j-k) h}+\delta_{(j+k) h}\right) \quad(1 \leqslant k \leqslant K),
$$

for a suitable parameter $K$, in order to economise on the number of operations required to obtain convergence of the iterative process.

In Table 1 we record associated exponents $\delta_{s}$ for $4 \leqslant s \leqslant 7.691$, rounded up in the final displayed decimal place. These values were calculated by using a step size of $h=0.005$, although we record only values at intervals of 0.1 . Since it is convenient in many circumstances to make use of admissible exponents $\Delta_{s}$, we record also in the table the values of $\Delta_{s}$ corresponding to each $\delta_{s}$, these being related by means of the formula

$$
\delta_{s}=\frac{1}{2} s-3+\Delta_{s} .
$$

We note that for $5<s<6$, for $6<s<6.5$, and for $7.365<s<7.691$, the associated exponents obtained by our calculations were simply linear interpolations between $\delta_{5}$ and $\delta_{6}$, between $\delta_{6}$ and $\delta_{6.5}$, and between $\delta_{7.365}$ and $\delta_{7.691}$, respectively. We omit intermediate values, therefore, in the interests of saving space. Of course, associated exponents may be derived for values of $s$ between those in the table by linear interpolation, such being justified by making use of the bounds stemming from convexity. Finally, we remark that Lemma 4.5 was required in the derivation of associated exponents $\delta_{s}$ only for $s>7.365$.

We conclude this section by noting that Theorem 2 follows immediately from Lemma 4.5 on taking $s=7.365$, for as is clear from Table 1 , one has

$$
7.691>7.365+10 \Delta_{7.365} \text {. }
$$

TABLE 1.

\begin{tabular}{lcc|ccc}
\hline$s$ & $\delta_{s}$ & $\Delta_{s}$ & \multicolumn{1}{c}{$s$} & $\delta_{s}$ & $\Delta_{s}$ \\
\hline 4.0 & 0.000000 & 1.000000 & 6.5 & 0.405318 & 0.155318 \\
4.1 & 0.001313 & 0.951313 & 6.6 & 0.438521 & 0.138521 \\
4.2 & 0.005005 & 0.905005 & 6.7 & 0.472029 & 0.122029 \\
4.3 & 0.010818 & 0.860818 & 6.8 & 0.505824 & 0.105824 \\
4.4 & 0.018282 & 0.818282 & 6.9 & 0.540259 & 0.090259 \\
4.5 & 0.026936 & 0.776936 & 7.0 & 0.576267 & 0.076267 \\
4.6 & 0.037983 & 0.737983 & 7.1 & 0.612863 & 0.062863 \\
4.7 & 0.049636 & 0.699636 & 7.2 & 0.650437 & 0.050437 \\
4.8 & 0.061881 & 0.661881 & 7.3 & 0.689361 & 0.039361 \\
4.9 & 0.074704 & 0.624704 & 7.365 & 0.715035 & 0.032535 \\
5.0 & 0.088092 & 0.588092 & 7.691 & 0.845500 & 0.000000 \\
6.0 & 0.249569 & 0.249569 & & & \\
\hline
\end{tabular}




\section{Waring's problem for cubes of smooth numbers}

The integers employed in the representation central to Theorem 1 are substantially smoother than is commonplace in modern treatments of Waring's problem, and this increases the difficulty of treating the major arc contribution in our application of the Hardy-Littlewood method. In order to circumvent technical difficulties of this type, we work with an artificial set similar to $\mathscr{A}(P, R)$. When $P, Q$ and $R$ are positive real numbers, we write

$$
\mathscr{A}^{*}(Q, R)=\{n \in[1, Q] \cap \mathbb{Z}: p \text { prime, } p \mid n \Rightarrow \sqrt{R}<p \leqslant R\},
$$

and then define the subset $\mathscr{C}(P, R)$ of $\mathscr{A}(P, R)$ by

$$
\mathscr{C}(P, R)=\left\{l m: m \in \mathscr{A}^{*}(P / \sqrt{R}, R) \text { and } 1 \leqslant l \leqslant \sqrt{R}\right\} .
$$

We note for future reference that each element of $\mathscr{C}(P, R)$ is uniquely represented in the form $l m$ described in the definition of this set, for such $l$ and $m$ are coprime. It is convenient also to define

$$
A^{*}(Q, R)=\operatorname{card}\left(\mathscr{A}^{*}(Q, R)\right) .
$$

Before proceeding further we require an estimate for $A^{*}(Q, R)$. Here and elsewhere in this section it is convenient to write

$$
L(x)=\exp (\sqrt{(\log x)(\log \log x)}) .
$$

Lemma 5.1. Let $c$ be a positive real number, and let $Q$ and $R$ be large real numbers with $R \geqslant L(Q)^{c}$. Then for each $\varepsilon>0$ one has

$$
A^{*}(Q, R) \gg Q L(Q)^{-1 / c-\varepsilon} \text {. }
$$

Proof. A simple counting argument suffices to provide a lower bound of the quality recorded in the statement of the lemma. Let the set of prime numbers $p$ satisfying $\sqrt{R}<p \leqslant R$ be $\mathscr{P}=\left\{p_{1}, p_{2}, \ldots, p_{t}\right\}$. Then as a consequence of the Prime Number Theorem, one has

$$
\frac{R}{2 \log R} \leqslant t \leqslant \frac{2 R}{\log R} .
$$

Next we observe that each element $n$ of $\mathscr{A}^{*}(Q, R)$ is uniquely represented in the form

$$
n=p_{1}^{a_{1}} p_{2}^{a_{2}} \ldots p_{t}^{a_{t}}
$$

with $p_{i} \in \mathscr{P}$ and $a_{i}$ a non-negative integer for $1 \leqslant i \leqslant t$. Let $\mathscr{T}$ denote the set of $t$-tuples of non-negative integers $\left(a_{1}, \ldots, a_{t}\right)$ satisfying the condition

$$
a_{1}+\ldots+a_{t} \leqslant \frac{\log Q}{\log R} .
$$

Then for each $\mathbf{a} \in \mathscr{T}$ one has

$$
a_{1} \log p_{1}+a_{2} \log p_{2}+\ldots+a_{t} \log p_{t} \leqslant \log Q,
$$

whence it follows from the uniqueness of the representation (5.2) that

$$
A^{*}(Q, R) \geqslant \operatorname{card}(\mathscr{T}) .
$$

The cardinality of the set $\mathscr{A}^{*}(Q, R)$ is plainly an increasing function of the 
parameter $R$. We therefore write

and suppose that

$$
m=\left[\frac{\log Q}{c \log L(Q)}\right]+1,
$$

$$
R=Q^{1 / m} \leqslant L(Q)^{c}
$$

In the latter circumstances one has $m=(\log Q) /(\log R)$. Also, by a well-known estimate (see, for example, Exercise 1 of Vaughan [11, Chapter 1]) one has

$$
\operatorname{card}(\mathscr{T})=(-1)^{t+1}\left(\begin{array}{c}
-(t+1) \\
m
\end{array}\right)=\frac{(t+m) !}{t ! m !} .
$$

On recalling (5.1), therefore, it follows from Stirling's formula that

$$
\begin{aligned}
\log (\operatorname{card}(\mathscr{T})) & \geqslant\left(m+t+\frac{1}{2}\right) \log (m+t)-\left(m+\frac{1}{2}\right) \log m-\left(t+\frac{1}{2}\right) \log t+O(1) \\
& >m \log (t / m)+m-\log m,
\end{aligned}
$$

whence

$$
\log (\operatorname{card}(\mathscr{T}))>m \log \left(\frac{R}{m \log R}\right)=\log Q-m \log \log Q .
$$

Then in view of (5.3), we obtain

$$
\log \left(A^{*}(Q, R)\right) \geqslant \log Q-\left(\frac{1}{c}+o(1)\right) \sqrt{(\log Q)(\log \log Q)},
$$

and the conclusion of the lemma follows immediately.

It is now possible to describe our strategy for proving Theorem 1. Let $c$ be a sufficiently large positive number, and let $n$ be an integer sufficiently large in terms of $c$. We take $R=L\left(n^{1 / 3}\right)^{c}$ and $P=n^{1 / 3} R^{1 / 800}$, and consider the number, $T(n)$, of representations of $n$ in the form

$$
n=x_{1}^{3}+x_{2}^{3}+\ldots+x_{8}^{3},
$$

with $x_{i} \in \mathscr{C}(P, R)(1 \leqslant i \leqslant 8)$. We aim to establish that for each positive number $\varepsilon$ one has

$$
T(n) \gg n^{5 / 3} L(P)^{-8 / c-\varepsilon},
$$

whence the conclusion of Theorem 1 follows immediately.

Define the exponential sum $h(\alpha)=h(\alpha ; P, R)$ by

$$
h(\alpha ; P, R)=\sum_{x \in \mathscr{C}(P, R)} e\left(\alpha x^{3}\right) .
$$

Then by orthogonality one has

$$
T(n)=\int_{0}^{1} h(\alpha)^{8} e(-\alpha n) d \alpha .
$$

We apply the Hardy-Littlewood method. Let

$$
Q=P^{1 / 3} \quad \text { and } Y=\sqrt{R},
$$

and write $\mathfrak{m}=\mathfrak{m}(Q), \mathfrak{M}=\mathfrak{M}(Q), \mathfrak{P}=\mathfrak{M}(Y)$ and $\mathfrak{p}=\mathfrak{m}(Y)$. As the first step in 
our analysis, we show that the contribution arising from the $\operatorname{arcs} \mathfrak{p}$ in (5.4) is of smaller order than the expected main term.

Lemma 5.2. One has

$$
\int_{\mathfrak{p}}|h(\alpha ; P, R)|^{8} d \alpha \ll P^{5} R^{-1 / 8} .
$$

Proof. We observe first that the computations described in $\S 4$ may be adapted to provide the estimate

$$
\int_{0}^{1}|h(\alpha)|^{s} d \alpha \ll P^{\mu_{s}+\varepsilon},
$$

with $s=7.365$ and $\mu_{s}=4.4$. In order to verify this assertion, one notes that

$$
\mathscr{C}(P, R) \subseteq \mathscr{A}(P, R),
$$

and moreover that the techniques of Wooley [14] embodied in Lemmata 4.1, 4.2 and 4.3 depend for their success, ultimately, only on estimates for the number of solutions of certain underlying diophantine equations. The substitution of the set $\mathscr{C}(P, R)$ here for the set $\mathscr{A}(P, R)$ in the latter work therefore has no deleterious effect on the desired bound embodied in (5.5). In view of (5.6), the argument applied in the proof of Lemma 2.2 to estimate $f(\alpha ; P, R)$ is also resilient to the substitution of the set $\mathscr{C}(P, R)$ for $\mathscr{A}(P, R)$. On recalling the relations (2.1), therefore, we find that when $\alpha \in \mathbb{R}$, and $a \in \mathbb{Z}$ and $q \in \mathbb{N}$ satisfy $(a, q)=1, q \leqslant Q$ and $|q \alpha-a| \leqslant Q P^{-3}$, then one has

$$
h(\alpha) \ll \frac{q^{\varepsilon-1 / 6} P(\log P)^{5 / 2+\varepsilon}}{\left(1+P^{3}|\alpha-a / q|\right)^{1 / 3}}+P^{9 / 10+\varepsilon} .
$$

Suppose that $\alpha \in \mathrm{m}$. By Dirichlet's Theorem on diophantine approximation, there exist $a \in \mathbb{Z}$ and $q \in \mathbb{N}$ with $(a, q)=1,1 \leqslant q \leqslant P^{2}$ and $|q \alpha-a| \leqslant P^{-2}$. On recalling the relations (2.1), we find from Lemma 2.2 that the upper bound (5.7) holds, whence $h(\alpha) \ll P^{9 / 10+\varepsilon}$, except possibly when $1 \leqslant q \leqslant P^{3 / 5}$ and $|q \alpha-a| \leqslant P^{-12 / 5}$. But in the latter circumstances, Lemma 2.2 again shows that (5.7) holds. Since $\alpha \in \mathfrak{m}$, moreover, one necessarily has $q+P^{3}|q \alpha-a|>Q$. On noting that we have established the inequality (5.7) free of the accompanying hypotheses, we may conclude that

$$
|h(\alpha)| \ll P^{17 / 18+\varepsilon},
$$

whence there exists a positive number $\tau$ with

$$
\begin{aligned}
\int_{\mathfrak{m}}|h(\alpha)|^{8} d \alpha & \ll\left(\sup _{\alpha \in \mathfrak{m}}|h(\alpha)|\right)^{8-s} \int_{0}^{1}|h(\alpha)|^{s} d \alpha \\
& \ll P^{\mu_{s}+(17 / 18)(8-s)+2 \varepsilon} \ll P^{5-\tau} .
\end{aligned}
$$

Next observe that the argument of the proof of Corollary 3.2 remains unaffected by the substitution of $h(\alpha ; P, R)$ for $f(\alpha ; P, R)$, whence whenever $1 \leqslant X \leqslant 2 P^{1 / 3}$ one has

$$
\int_{\mathfrak{M}(X)}|h(\alpha)|^{6} d \alpha \ll X^{\varepsilon} P^{3}(\log P)^{10+\varepsilon} .
$$


But in view of (5.7), whenever $Y \leqslant X \leqslant P^{1 / 3}$, one has

$$
\sup _{\alpha \in \mathfrak{N}(X)}|h(\alpha)| \ll P X^{-1 / 7} .
$$

It therefore follows from (5.9) that in such circumstances one has

$$
\begin{aligned}
\int_{\mathfrak{N}(X)}|h(\alpha)|^{8} d \alpha & \ll\left(\sup _{\alpha \in \mathfrak{N}(X)}|h(\alpha)|\right)^{2} \int_{\mathfrak{M}(2 X)}|h(\alpha)|^{6} d \alpha \\
& \ll\left(P X^{-1 / 7}\right)^{2} X^{\varepsilon} P^{3}(\log P)^{10+\varepsilon} \ll P^{5} X^{-1 / 4} .
\end{aligned}
$$

On summing over dyadic intervals, therefore, we may conclude that

whence by (5.8),

$$
\int_{\mathfrak{M} \backslash \mathfrak{P}}|h(\alpha)|^{8} d \alpha \ll P^{5} Y^{-1 / 4},
$$

$$
\int_{\mathfrak{p}}|h(\alpha)|^{8} d \alpha=\int_{\mathfrak{m}}|h(\alpha)|^{8} d \alpha+\int_{\mathfrak{M} \backslash \mathfrak{P}}|h(\alpha)|^{8} d \alpha \ll P^{5} Y^{-1 / 4} .
$$

The conclusion of the lemma follows immediately.

In order to investigate the contribution arising from the arcs $\mathfrak{P}$ we must provide a suitable major arc approximation to the function $h(\alpha)$. In order to describe this approximation, we write

$$
S(q, a)=\sum_{r=1}^{q} e\left(a r^{3} / q\right) \quad \text { and } \quad v(\beta ; Z)=\int_{0}^{Z} e\left(\beta \gamma^{3}\right) d \gamma .
$$

Lemma 5.3. Suppose that $\alpha \in \mathfrak{M}(q, a ; Y) \subseteq \mathfrak{P}$. Then

$$
h(\alpha ; P, R)=q^{-1} S(q, a) \sum_{m \in \mathscr{A}^{*}(P / \sqrt{R}, R)} v\left((\alpha-a / q) m^{3} ; \sqrt{R}\right)+O\left(P R^{\varepsilon-1 / 4}\right) .
$$

Proof. Suppose that $\alpha \in \mathfrak{M}(q, a ; Y) \subseteq \mathfrak{P}$, so that $a \in \mathbb{Z}, q \in \mathbb{N},(a, q)=1$, $q \leqslant Y$ and $|q \alpha-a| \leqslant Y P^{-3}$. Since each of the prime divisors of any element of $\mathscr{A}^{*}(P / \sqrt{R}, R)$ exceeds $Y=\sqrt{R}$, it follows that for each $m \in \mathscr{A}^{*}(P / \sqrt{R}, R)$ one has $(m, q)=1$. Thus we deduce from Vaughan [11, Theorem 4.1] that for each $m \in \mathscr{A}^{*}(P / \sqrt{R}, R)$ one has

$$
\begin{aligned}
\sum_{1 \leqslant x \leqslant \sqrt{R}} e\left(\alpha m^{3} x^{3}\right) & =q^{-1} S\left(q, a m^{3}\right) v\left((\alpha-a / q) m^{3} ; \sqrt{R}\right)+O\left(Y^{1 / 2+\varepsilon}\right) \\
& =q^{-1} S(q, a) v\left((\alpha-a / q) m^{3} ; \sqrt{R}\right)+O\left(R^{1 / 4+\varepsilon}\right) .
\end{aligned}
$$

The conclusion of the lemma follows immediately on summing over the elements of $\mathscr{A}^{*}(P / \sqrt{R}, R)$.

We are now poised to complete the proof of Theorem 1. Define the function $h^{*}(\alpha)=h^{*}(\alpha ; P, R)$ for $\alpha \in \mathfrak{P}$ by taking

$$
h^{*}(\alpha ; P, R)=q^{-1} S(q, a) \sum_{m \in \mathscr{A}^{*}(P / \sqrt{R}, R)} v\left((\alpha-a / q) m^{3} ; \sqrt{R}\right),
$$


when $\alpha \in \mathfrak{M}(q, a ; Y) \subseteq \mathfrak{P}$. Then it follows from Lemma 5.3 that

$$
\sup _{\alpha \in \mathfrak{\beta}}\left|h(\alpha)-h^{*}(\alpha)\right| \ll P R^{\varepsilon-1 / 4},
$$

whence for $\alpha \in \mathfrak{P}$ one has

$$
\begin{aligned}
\left|h(\alpha)^{8}-h^{*}(\alpha)^{8}\right| & \ll\left|h(\alpha)-h^{*}(\alpha)\right|\left(|h(\alpha)|^{7}+\left|h(\alpha)-h^{*}(\alpha)\right|^{7}\right) \\
& \ll P^{2} R^{\varepsilon-1 / 4}|h(\alpha)|^{6}+P^{8} R^{8 \varepsilon-2} .
\end{aligned}
$$

On recalling (5.9) and noting that the measure of $\mathfrak{P}$ is $O\left(R P^{-3}\right)$, therefore, one finds that

$$
\begin{aligned}
\int_{\mathfrak{P}} h(\alpha)^{8} e(-\alpha n) d \alpha-\int_{\mathfrak{P}} h^{*}(\alpha)^{8} e(-\alpha n) d \alpha \\
\ll P^{5} R^{8 \varepsilon-1}+P^{5}(\log P)^{10+\varepsilon} R^{2 \varepsilon-1 / 4},
\end{aligned}
$$

whence by Lemma 5.2,

$$
\int_{0}^{1} h(\alpha)^{8} e(-\alpha n) d \alpha-\int_{\mathfrak{P}} h^{*}(\alpha)^{8} e(-\alpha n) d \alpha \ll P^{5} R^{-1 / 8} .
$$

Next we observe that by a change of variable, one obtains

$$
\sum_{m \in \mathscr{A}^{*}(P / \sqrt{R}, R)} v\left(\beta m^{3} ; \sqrt{R}\right)=\sum_{m \in \mathscr{A}^{*}(P / \sqrt{R}, R)} \frac{1}{m} \int_{0}^{m \sqrt{R}} e\left(\beta \gamma^{3}\right) d \gamma,
$$

and thus, by partial integration,

$$
\sum_{m \in \mathscr{A}^{*}(P / \sqrt{R}, R)} v\left(\beta m^{3} ; \sqrt{R}\right) \ll \frac{P \log P}{\left(1+P^{3}|\beta|\right)^{1 / 3}} .
$$

On recalling (5.10), therefore, we find that the standard end-game technique (see, for example, Chapter 4 of Vaughan [11]) now reveals that

$$
\int_{\mathfrak{P}} h^{*}(\alpha)^{8} e(-\alpha n) d \alpha-\subseteq(n) J(n) \ll P^{5}(\log P)^{8} Y^{-2 / 3} \ll P^{5} R^{-1 / 8},
$$

where we write

$$
\mathfrak{S}(n)=\sum_{q=1}^{\infty} \sum_{\substack{a=1 \\(a, q)=1}}^{q}\left(q^{-1} S(q, a)\right)^{8} e(-n a / q),
$$

$$
J(n)=\sum_{m_{1}, \ldots, m_{8} \in \mathscr{A}^{*}(P / \sqrt{R}, R)}\left(m_{1} \ldots m_{8}\right)^{-1} \int_{-\infty}^{\infty} \int_{\mathscr{B}(\mathbf{m})} e\left(\beta\left(\gamma_{1}^{3}+\ldots+\gamma_{8}^{3}-n\right)\right) d \gamma d \beta,
$$

and the box $\mathscr{B}(\mathbf{m})$ is defined by

$$
\mathscr{B}(\mathbf{m})=\left[0, m_{1} \sqrt{R}\right] \times\left[0, m_{2} \sqrt{R}\right] \times \ldots \times\left[0, m_{8} \sqrt{R}\right] .
$$

The singular series $\mathfrak{S}(n)$ is the familiar one associated with Waring's problem for sums of eight cubes, and thus one has $\widetilde{S}(n) \gg 1$ uniformly in $n$. Also, on 
combining Lemma 5.1 with a trivial estimate, we obtain

$$
\begin{aligned}
\sqrt{R}\left(A^{*}(P / \sqrt{R}, R)-A^{*}\left(\frac{2}{3} n^{1 / 3} / \sqrt{R}, R\right)\right) & \gg P L(P)^{-1 / c-\varepsilon}+O\left(P R^{-1 / 800}\right) \\
& \gg P L(P)^{-1 / c-\varepsilon},
\end{aligned}
$$

provided that $c>800$. But whenever $m_{i} \geqslant \frac{2}{3} n^{1 / 3} / \sqrt{R}$ for $1 \leqslant i \leqslant 8$, one has $\left[0, \frac{2}{3} n^{1 / 3}\right]^{8} \subseteq \mathscr{B}(\mathbf{m})$, and thus an application of Fourier's integral formula rapidly establishes that

$$
\begin{aligned}
J(n) \gg & \sum_{\substack{\left(2 n^{1 / 3}\right) /(3 \sqrt{R})<m_{1} \leqslant P / \sqrt{R} \\
m_{1} \in \mathscr{A}^{*}(P / \sqrt{R}, R)}} \ldots \sum_{\substack{\left(2 n^{1 / 3}\right) /(3 \sqrt{R})<m_{8} \leqslant P / \sqrt{R} \\
m_{8} \in \mathscr{A}^{*}(P / \sqrt{R}, R)}} n^{5 / 3}\left(m_{1} \ldots m_{8}\right)^{-1} \\
& \gg n^{5 / 3}(P / \sqrt{R})^{-8}\left(\frac{P}{\sqrt{R}} L(P)^{-1 / c-\varepsilon}\right)^{8} .
\end{aligned}
$$

On recalling (5.4), (5.11) and (5.13), therefore, we may finally conclude that

$$
T(n) \gg n^{5 / 3} L(P)^{-8 / c-8 \varepsilon}+O\left(n^{5 / 3} L(P)^{-c / 20}\right),
$$

and thus Theorem 1 follows whenever $c$ is sufficiently large.

Observe that the methods concluding the proof of Theorem 1 above together with those of Chapter 4 of Vaughan [11], may be used to show that, uniformly for $\frac{1}{2} n<m \leqslant n$, one has the lower bound

$$
\int_{\mathfrak{P}} h(\alpha)^{4} e(-\alpha m) d \alpha \gg n^{1 / 3}(P / \sqrt{R})^{-4}\left(A^{*}(P / \sqrt{R}, R)\right)^{4} .
$$

On combining the latter bound, through the medium of Bessel's inequality, with the minor arc bound provided by Lemma 5.2, one may routinely establish that almost all positive integers are the sum of four positive integral smooth cubes. Let $E(X ; R)$ denote the number of integers $n$ with $1 \leqslant n \leqslant X$ for which the diophantine equation

$$
n=x_{1}^{3}+x_{2}^{3}+x_{3}^{3}+x_{4}^{3}
$$

has no solution with $P\left(x_{i}\right) \leqslant R(1 \leqslant i \leqslant 4)$. The reader may verify that a careful analysis along the above lines shows that when $\eta$ is a sufficiently small positive number and $X$ is a sufficiently large real number, then whenever $L(X)^{c} \leqslant R \leqslant X^{\eta}$, one has

$$
E(X ; R) \ll_{\varepsilon} X R^{\varepsilon-1 / 6} .
$$

\section{References}

1. R. C. Baker, J. Brüdern and T. D. Wooley, 'Cubic diophantine inequalities', Mathematika 42 (1995) 264-277.

2. A. BALOG and J. BRÜDERN, 'Sums of three cubes in three linked three-progressions', J. Reine Angew. Math. 466 (1995) 45-85.

3. J. BRÜDERN, 'A sieve approach to the Waring-Goldbach problem II. On the seven cubes theorem', Acta Arith. 72 (1995) 211-227.

4. J. BRÜDERN and T. D. WOOLEY, 'On Waring's problem: a square, four cubes and a biquadrate', Math. Proc. Cambridge Philos. Soc. 127 (1999) 193-200.

5. G. Harcos, 'Waring's problem with small prime factors', Acta Arith. 80 (1997) 165-185.

6. L.-K. HuA, Additive theory of prime numbers (American Mathematical Society, Providence, RI, 1965). 
7. Ju V. Linnik, 'On the representation of large numbers as sums of seven cubes', Mat. Sb. 12 (1943) 218-224.

8. G. Tenenbaum, Introduction to analytic and probabilistic number theory (Cambridge University Press, 1995).

9. R. C. Vaughan, 'On Waring's problem for cubes', J. Reine Angew. Math. 365 (1986) 122-170.

10. R. C. Vaughan, 'A new iterative method in Waring's problem', Acta. Math. 162 (1989) 1-71.

11. R. C. Vaughan, The Hardy-Littlewood method (Cambridge University Press, 1997).

12. R. C. Vaughan and T. D. Wooley, 'On Waring's problem: some refinements', Proc. London Math. Soc. (3) 63 (1991) 35-68.

13. T. D. Wooley, 'Large improvements in Waring's problem', Ann. of Math. 135 (1992) 131-164

14. T. D. Wooley, 'Breaking classical convexity in Waring's problem: sums of cubes and quasidiagonal behaviour', Invent. Math. 122 (1995) 421-451.

\section{Jörg Brüdern}

Mathematisches Institut A

Universität Stuttgart

Postfach 801140

D-70511 Stuttgart

Germany

bruedern@mathematik.uni-stuttgart.de
Trevor D. Wooley

Department of Mathematics University of Michigan

East Hall

525 East University Avenue

Ann Arbor

MI 48109-1109

USA

wooley@math.1sa.umich.edu 